\title{
The influence of short-time ball-milling on the stability of amorphous $\mathrm{CoFeB}$ alloys
}

\author{
K Saksl $^{1,5}, \mathbf{J}$ Bednarčík ${ }^{1,2,3,5}, \mathbf{R}$ Nicula $^{2}$, E Burkel $^{2}$, S Roth $^{4}$ and H Franz $^{1}$ \\ ${ }^{1}$ HASYLAB am Deutschen Elektronen Synchrotron, DESY, Notkestrasse 85, D-22603 Hamburg, \\ Germany \\ ${ }^{2}$ Institute of Physics, Rostock University, August-Bebel-Strasse 55, D-18055 Rostock, Germany \\ ${ }^{3}$ Institute of Physics, Faculty of Science, P J Šafárik University, Park Angelinum 9, \\ 04154 Košice, Slovakia \\ ${ }^{4}$ IFW Dresden, Institut für Metallische Werkstoffe, Helmholtzstraße 20, D-01069 Dresden, \\ Germany \\ E-mail: karel.saksl@desy.de and jozef.bednarcik@upjs.sk
}

Received 28 August 2006, in final form 27 February 2007

Published 30 March 2007

Online at stacks.iop.org/JPhysCM/19/176215

\begin{abstract}
The influence of short-time milling on the atomic structure of amorphous $\mathrm{Co}_{70.3} \mathrm{Fe}_{4.7} \mathrm{~B}_{25}$ has been investigated by differential scanning calorimetry (DSC), x-ray powder diffraction (XRD), vibrating sample magnetometer (VSM) and $\mathrm{x}$-ray absorption fine-structure (XAFS) techniques. Our results prove that the milling process crystallizes the initially amorphous sample and that the degree of inherent crystallization is inversely proportional to the powder particle size. The investigation of the local atomic structure documents very similar environments around the $\mathrm{Co}$ and $\mathrm{Fe}$ atoms. The high-energy ball-milling of amorphous precursor represents a practical way to prepare powders having the desired amorphous/nanocrystalline microstructure.
\end{abstract}

\section{Introduction}

Amorphous and nanocrystalline ferromagnetic alloys possess excellent soft-magnetic properties, challenging researchers aiming to extend their technical exploitation [1]. Melt spinning, splat quenching and condensation from the gas phase are nowadays well established techniques and employed for preparation of amorphous, particularly soft-magnetic amorphous, materials [2]. The main drawback of all these synthesis methods is their limitation in complex shaping of bulk samples, nowadays constrained to films or ribbons. It is therefore necessary to attempt preparation of such materials in bulk form, for example as cylinders or rings, that would be more convenient for industrial applications. Powder metallurgy represents an alternative way of producing bulk and, at the same time, amorphous soft magnetic materials possessing

5 Authors to whom any correspondence should be addressed. 
the required shape [3]. There is the rational assumption that non-magnetostrictive alloys are suitable for the preparation of bulk samples by compaction of powders prepared by ball-milling. When magnetostriction is low (preferably zero), the magnetic properties of such near-zero magnetostrictive materials are insensitive to mechanical stress because the effect of magnetomechanical coupling is in this case suppressed. On the other hand, magnetic properties of stress-sensitive (non-zero magnetostrictive) materials are considerably degraded even by small stresses. Such materials must therefore be carefully annealed after the final forming step.

The structural study discussed in this paper is part of our work focused on development and optimization of magnetic properties of Co-rich bulk metallic glasses. Our primary goal is to combine the high permeability, zero magnetostriction and low coercivity of Co-rich amorphous alloys [4] to create a bulk material possessing good mechanical properties. The synthesis procedure starts with the preparation of amorphous powder precursors by ball-milling of either melt-spun ribbons or elemental powders, followed by consolidation using hot pressing.

In the present report we focus only on the first preparation step, i.e. to determine the structural and magnetic properties of powders obtained by ball-milling of Co-rich melt-spun ribbons. Special emphasis was devoted to the investigation of the influence of short-time ballmilling on the stability of the amorphous phase. In our studies we focus on the amorphous $\mathrm{Co}_{70.3} \mathrm{Fe}_{4.7} \mathrm{~B}_{25}$ alloy. This alloy has a balanced concentration of $\mathrm{Co}, \mathrm{Fe}$, and metalloid, and could be in principle non-magnetostrictive (for example, amorphous $\mathrm{Co}_{70.5} \mathrm{Fe}_{4.5} \mathrm{Si}_{10} \mathrm{~B}_{15}$ alloy has zero magnetostriction $[4,5])$.

\section{Experimental details}

\subsection{Sample preparation}

The master alloy with nominal composition $\mathrm{Co}_{70.3} \mathrm{Fe}_{4.7} \mathrm{~B}_{25}$ (at.\%) was prepared by arc-melting of high-purity elements (>99.8\%) under argon atmosphere. Ribbons of $10 \mathrm{~mm}$ width and $\sim 40 \mu \mathrm{m}$ thickness have been prepared from this pre-alloy by single-roller melt-spinning. Some of the ribbons was in the next step milled for $2 \mathrm{~h}$ in a Retsch PM4000 planetary ball mill with steel vials and balls. The milling experiment was performed at a ball-to-powder mass ratio of 6:1 with a speed of $200 \mathrm{rpm}$. To ensure effective milling, the direction of rotation of the milling plate was changed every minute. All powder handling was carried out in a glove box under argon atmosphere (level of $\mathrm{O}_{2}, \mathrm{H}_{2} \mathrm{O}<1 \mathrm{ppm}$ ). The powder samples obtained by the milling were further sieved in a Retsch sieve shaker equipped with a series of sieves with mesh sizes 500, 250, 125, 63, 45 and $20 \mu \mathrm{m}$.

The structure of the as-quenched ribbon and the powders with fractioned particle sizes $<20(\mathrm{~N} 1), 20-45(\mathrm{~N} 2), 45-63(\mathrm{~N} 3)$ and $>63 \mu \mathrm{m}(\mathrm{N} 4)$ were further examined by DSC, XRD, VSM and XAFS techniques.

\subsection{DSC, XRD, VSM and XAFS measurements}

Thermal analysis was performed using a differential scanning calorimeter (Netzsch DSC 404) at a heating rate of $0.33 \mathrm{~K} \mathrm{~s}^{-1}$ under vacuum.

High-energy x-ray powder diffraction measurements were performed at HASYLAB at DESY (Hamburg, Germany) at the PETRA2 experimental station located at the PETRA electron storage ring operating at electron energy of $11.28 \mathrm{GeV}$ and a stored current in the range of 40-10 $\mathrm{mA}$. The samples measured at room temperature in transmission mode were illuminated for $60 \mathrm{~s}$ by a well collimated $1 \mathrm{~mm}^{2}$ incident beam of photon energy $110 \mathrm{keV}$ $(\lambda=0.1127 \AA$ ). The corresponding XRD patterns were recorded by a 2D detector (mar345 
image plate) in asymmetric mode to obtain data at high magnitude of wavevector transfer $Q$. The background intensity was subtracted directly from the 2D XRD pattern and the result was integrated to $Q$-space by using the program Fit2D [6]. The integrated data were corrected for polarization, fluorescence and inelastic scattering and the total structural factor $S(Q)$ was obtained by using the Faber-Ziman equation [7].

The saturation magnetization $M_{\mathrm{s}}$ and coercivity $H_{\mathrm{c}}$ of the as-quenched ribbon and sieved powder samples $(\mathrm{N} 1, \mathrm{~N} 2, \mathrm{~N} 3$ and N4) were determined from magnetization loops traced in a magnetic field with maximum induction of $\mu_{0} H=1.9 \mathrm{~T}$ using the vibrating sample magnetometer VSM-LakeShore 735 . The coercivity $H_{\mathrm{c}}$ of the as-quenched ribbon, on the other hand, was determined from the magnetization loop measured with a DC-hysteresis graph. All magnetic measurements were performed at room temperature.

X-ray absorption fine-structure (XAFS) measurements were performed at beamline E4 located at the DORIS III positron storage ring (energy $4.45 \mathrm{GeV}$, current $140-100 \mathrm{~mA}$ ) at HASYLAB. Data were collected in transmission mode at the $\mathrm{Fe}$ and Co $\mathrm{K}$ edge using a fixed exit double-crystal $\mathrm{Si}(111)$ monochromator. The $\mathrm{X}$-ray intensities were monitored using ionization chambers filled by gases, the type and pressure of which were adjusted to the corresponding energies. The energy calibration for Co and Fe was monitored using reference materials measured together with the sample. Experimentally measured x-ray absorption cross sections $\mu(E) x$ were analysed by the FEFF 8.1 (program for ab initio multiplescattering calculations of $\mathrm{x}$-ray absorption fine structure) [8] and Viper (program for XAFS data processing and refinement) codes [9].

\section{Theoretical background}

\subsection{Calculation of $x$-ray structure factor and pair correlation function from $x$-ray powder diffraction $(X R D)$ patterns}

According to Faber and Ziman [7] the total structure factor, $S(Q)$, can be obtained from the normalized elastically scattered intensity, $I_{\mathrm{e}}(Q)$, as

$$
S(Q)=\frac{I_{\mathrm{e}}(Q)-\left\langle f^{2}(Q)\right\rangle}{\langle f(Q)\rangle^{2}}
$$

with

$$
\langle f(Q)\rangle^{2}=\left(\sum_{i} c_{i} f_{i}(Q)\right)^{2}, \quad\left\langle f^{2}(Q)\right\rangle=\sum_{i} c_{i} f_{i}^{2}(Q),
$$

momentum transfer $Q=4 \pi \sin (\theta) / \lambda, c_{i}$ the concentration of atoms of type $i$ and $f_{i}(Q)=$ $f_{0}(Q)+f^{\prime}(\lambda)+f^{\prime \prime}(\lambda)$ the atomic scattering factor of element $i$, modified by the anomalous dispersion terms $f^{\prime}(\lambda)$ and $f^{\prime \prime}(\lambda)$ depending on the radiation wavelength. From the $S(Q)$ an atomic pair correlation (distribution) function, $G(r)$, can be calculated by means of a sine Fourier transform as

$$
G(r)=\frac{\rho(r)}{\rho_{0}}=1+\frac{1}{2 \pi^{2} r \rho_{0}} \int_{Q \min }^{Q \max } Q(S(Q)-1) \sin (Q r) \mathrm{d} Q
$$

here $\rho(r)$ and $\rho_{0}$ are the local and average atomic number density, respectively.

The average coordination number $N$, around any given atom in a spherical shell determined by distances $r_{1}$ and $r_{2}$, can then be calculated as

$$
N=\int_{r_{1}}^{r_{2}} 4 \pi \rho_{0} r^{2} G(r) \mathrm{d} r
$$




\subsection{Near-edge $x$-ray absorption fine-structure (XANES) calculation}

The near-edge features (50-100 eV above the absorption edge) reflect the local electron density of states close to the Fermi level. The signal provides information about local symmetry and local network topology so that, in principle, the XANES analysis could allow us to recover the detailed 3D local structure around the absorber [10]. There are two theoretical methods available for calculation of x-ray absorption spectra: (1) the band structure theory [11] and (2) the real space multiple-scattering (RSMS) theory [8, 12, 13]. Among the RSMS codes designed for XANES calculations for a given atomic cluster the most widely used is the FEFF software [8]. In this code a local structure around the absorber is assumed, and on its basis the potential acting on the photoelectron is calculated in the muffin-tin approximation and then used to solve the Schrödinger equation in the multiple-scattering formalism; finally, the absorption cross section is evaluated. This procedure produced very good results in many different systems.

In this paper we present a qualitative comparison of XANES signals measured at Co and Fe K edges with theory curves calculated using the FEFF 8.1 program. As input for our RSMS calculation we chose $\mathrm{Co}_{2} \mathrm{~B}$ coordination clusters modelled around $\mathrm{Co}$ or $\mathrm{Fe}$ absorbing atoms. Signals from three different cluster bodies (diameter 6, 8 and $11.4 \AA$ containing 15, 29 and 94 atoms, respectively) were calculated in order to demonstrate the minimum volume capable of reconstructing major features of the measured XANES resonances.

\subsection{Refinement of local structure parameters from extended $x$-ray absorption fine structure (EXAFS)}

EXAFS is usually referred to the oscillating part of the XAFS signal between $\sim 70$ and $1000 \mathrm{eV}$ above an absorption edge of a particular element of a sample. The signal contains structural information about short-range order around a specific type of atom, and its analysis provides a practical way to study the local atomic arrangement in materials.

The EXAFS signal $\chi(k)$ measured above the Co and Fe K edge was first extracted and weighted by $k^{2}$. The region where the non-weighted amplitude of the oscillatory signal $\chi(k)$ still dominates over the noise (for details see [9]) was Fourier transformed $(F T)$ to real space. The obtained $F T$ presents an equivalent to the pair correlation function $G(r)$, equation (3), but its signal is modified by phase shifts due to the photoelectron emission and back-scattering process. In the next step the main peak from the $F T$ was filtered (using a Hanning window function, 0-2.9 $\AA$, coefficient $A=0.01$ ) and back-transformed to $k$ space.

In order to derive quantitative values for the interatomic distances $R$, coordination numbers $N$, mean-square relative displacements $\sigma^{2}$ and threshold energy shifts $E_{0}$ one needs to carry out a numerical curve fitting analysis of $k$-space amplitudes and phases by following the EXAFS formula based on the single-scattering approximation [14]:

$$
k^{2} \chi(k)=k^{2} \sum_{i} C_{i}(k) \sin \left[2 k R_{i}+\varphi_{i}(k)\right] .
$$

The summation extends over $i$ coordination shells at average distance $R_{i}$ from the absorbing atom. $\varphi_{i}(k)$ is the total phase shift due to contributions from both the absorbing and the backscattering atom and $k$ is the photo-electron wavevector. The conversion between wavevector and energy is $k=\sqrt{\frac{2 m}{\hbar^{2}}\left(E-E_{0}\right)}$; here $m$ is the mass of an electron, $\hbar=\frac{h}{2 \pi}$ is Planck's constant, $E$ is the incident photon energy and $E_{0}$ is the threshold energy of this particular absorption edge. The amplitude factor $C_{i}(k)$ is given by

$$
C_{i}(k)=\frac{N_{i}}{k R_{i}^{2}} S_{0}^{2} F_{i}(k) \exp \left(-2 \sigma_{i}^{2} k^{2}\right),
$$




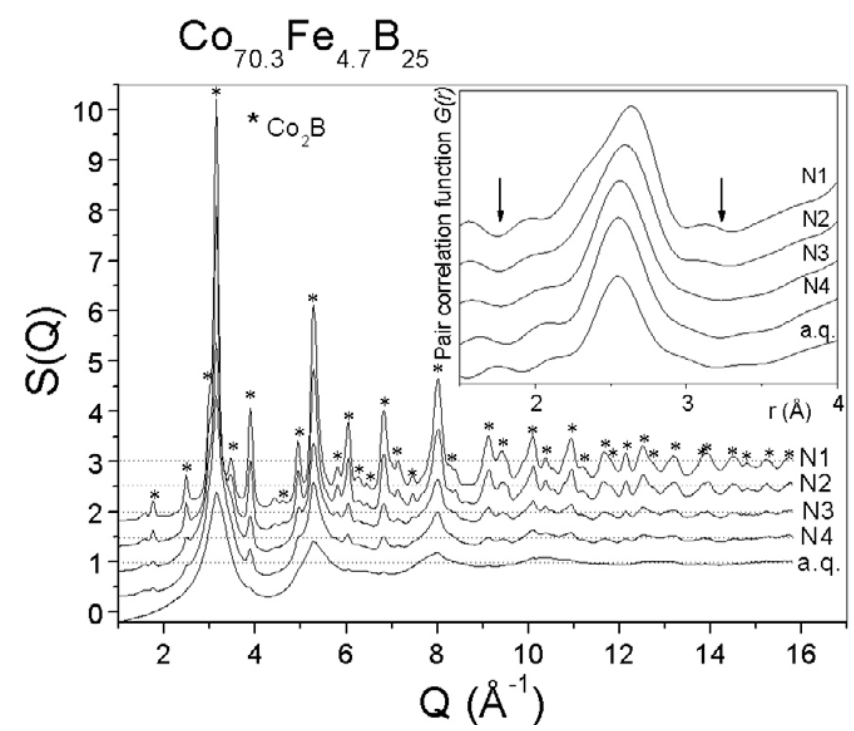

Figure 1. Structure factors, $S(Q)$, of the $\mathrm{CO}_{70.3} \mathrm{Fe}_{4.7} \mathrm{~B}_{25}$ alloy; a.q.- -as-quenched (ribbon) sample and sieved powder samples of grain size fractions $<20(\mathrm{~N} 1), 20-45(\mathrm{~N} 2), 45-63(\mathrm{~N} 3)$ and $>63 \mu \mathrm{m}$ (N4). The inset of the figure shows the pair correlation functions $G(r)$ of the first atomic coordination shell.

where $N_{i}$ is the average number of scattering atoms (coordination number), $S_{0}^{2}$ is a energyindependent many-body amplitude reduction factor that accounts for losses only within the central absorbing atom and is independent of the chemical nature and type of back-scattering atoms, $F_{i}(k)$ is the back-scattering amplitude characteristic of a particular type of backscattering atom and $\sigma_{i}^{2}$ is the mean square relative displacement. Expressions (5) and (6) are implemented in the program Viper for EXAFS data processing and refinement. The backscattering amplitude $F_{i}(k)$, the phase shift $\varphi_{i}(k)$ and the many-body amplitude reduction factors $S_{0}^{2}$ were calculated from the atomic configuration using the FEFF 8.1 code (the same clusters as for XANES calculation).

\section{Results and discussion}

\subsection{XRD measurements}

Figure 1 shows total structure factors $S(Q)$ from the as-quenched $\mathrm{Co}_{70.3} \mathrm{Fe}_{4.7} \mathrm{~B}_{25}$ alloy and the milled powder samples. The structure of the as-quenched sample is not fully amorphous, its $S(Q)$ showing tiny Bragg peaks visible on top of the broad diffuse contribution from the amorphous phase. Unlike the as-quenched sample the signal from the powder samples shows significantly different patterns, consisting of regular peaks, the intensity of which is increasing with decreasing powder particle sizes. So the smaller powder particles have a higher degree of crystallinity. Our previous studies also demonstrate that mechanically induced crystallization of $\mathrm{Co}_{70.3} \mathrm{Fe}_{4.7} \mathrm{~B}_{25}$ alloy is the most plausible scenario for observed transformation and can be retarded when milling is conducted at temperature of liquid nitrogen, $77 \mathrm{~K}$ (so called cryomilling) [15]. The nanocrystalline phase identified in all the samples is tetragonal $\mathrm{Co}_{2} \mathrm{~B}-$ like (SG $I 4 / \mathrm{mcm}$ ). The lattice parameters $a=5.06(2)$ and $c=4.19$ (7) $\AA$, determined by the full pattern Rietveld analysis, are slightly different from the $a=5.015$ and $c=4.220 \AA$ 


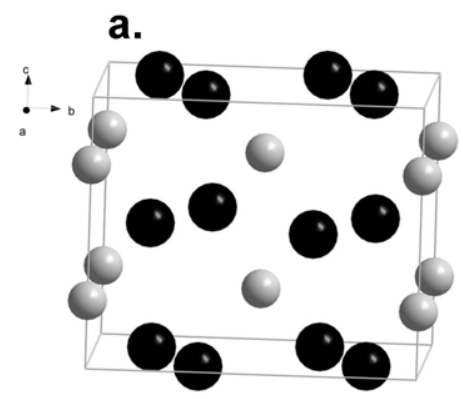

b.

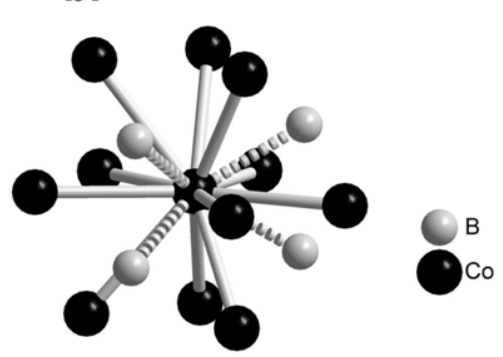

Figure 2. Schematic drawings of (a) the tetragonal $\mathrm{Co}_{2} \mathrm{~B}$ phase (PDF no 25-241; $\mathrm{SG} \mathrm{I4} / \mathrm{mcm}$, $a=5.06(2)$ and $c=4.19(7) \AA$ ) and (b) the first coordination shell around a Co or Fe atom; diameter of the cluster $6 \AA$.

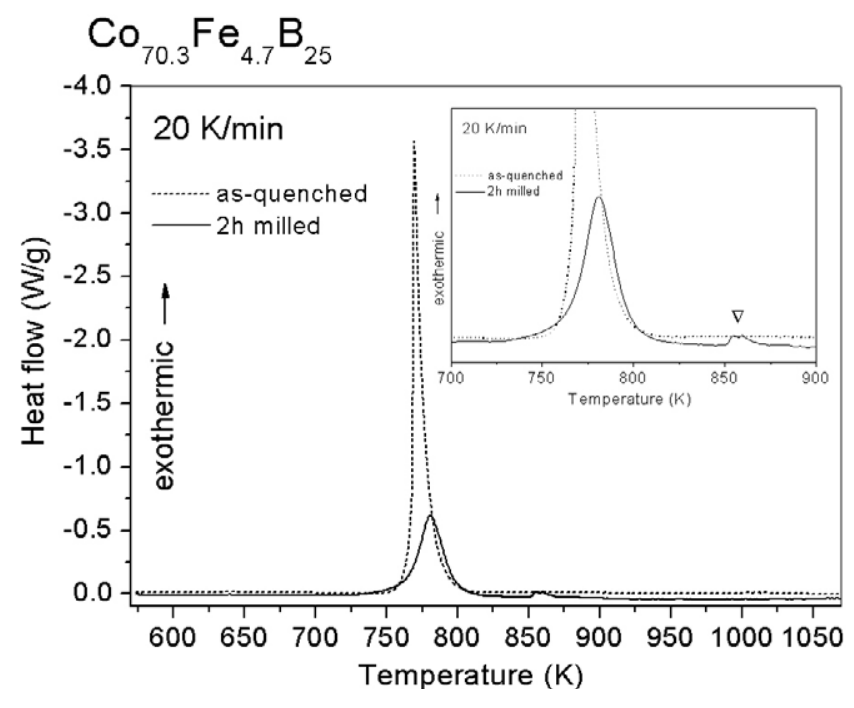

Figure 3. DSC scanning curves of the as-quenched (ribbon) and milled (powder) $\mathrm{Co}_{70.3} \mathrm{Fe}_{4.7} \mathrm{~B}_{25}$ alloy obtained at a heating rate of $0.33 \mathrm{~K} \mathrm{~s}^{-1}$. The inset of the figure shows the crystallization region. The open triangle marks the second crystallization peak observed only on the powder sample.

reported for pure $\mathrm{Co}_{2} \mathrm{~B}$ phase (PDF no 25-241) [16]. A schematic drawing of the $\mathrm{Co}_{2} \mathrm{~B}$ phase is shown in figure 2(a). The inset of figure 1 shows the pair correlation functions $G(r)$ calculated from the corresponding $S(Q)$. Closer inspection of the figure reveals a progressive change in the first shell (shoulder on the left from the maximum, peak $\sim 3.2 \AA$ ) depending on the degree of sample crystallization. The total number of atoms in the first shell (region marked by arrows) of the as-quenched and powder samples was calculated to be 13.7 and $15.0 \pm 0.1$ atoms, respectively.

\subsection{DSC measurements}

Figure 3 shows two DSC curves from $\mathrm{Co}_{70.3} \mathrm{Fe}_{4.7} \mathrm{~B}_{25}$. The dashed line represents the calorimetric signal from an as-quenched sample while the full line originates from the same alloy but milled for $2 \mathrm{~h}$ (powder sample before sieving). Comparison of these two curves brought several interesting observations. 
Table 1. Coercivity $H_{\mathrm{c}}$ and saturation magnetization $M_{\mathrm{S}}$ of as-quenched ribbon and powder $\mathrm{Co}_{70.3} \mathrm{Fe}_{4.7} \mathrm{~B}_{25}$ alloy determined from hysteresis loops traced at room temperature using a DChysteresis graph and VSM.

\begin{tabular}{lcl}
\hline Sample & $H_{\mathrm{c}}\left(\mathrm{A} \mathrm{m}^{-1}\right)$ & $M_{\mathrm{s}}\left(\mathrm{A} \mathrm{m}^{2} \mathrm{~kg}^{-1}\right)$ \\
\hline As-quenched & $19 \pm 1$ & $148.5 \pm 1$ \\
$\mathrm{~N} 1$ & $10320 \pm 100$ & $105.8 \pm 1$ \\
$\mathrm{~N} 2$ & $5110 \pm 100$ & $104.8 \pm 1$ \\
$\mathrm{~N} 3$ & $3180 \pm 100$ & $103.7 \pm 1$ \\
N4 & $2290 \pm 100$ & $103.8 \pm 1$ \\
\hline
\end{tabular}

Both records do not show a glass transitions $T_{\mathrm{g}}$ (a small endothermic hump) prior to the crystallization peak. Although it is generally accepted that the glass transition is a characteristic feature of amorphous material, several alloys capable of forming a stable amorphous phase, e.g. Al-based metallic glasses $[17,18]$, do not show this feature at all.

According to the $\mathrm{x}$-ray data, $\mathrm{Co}_{2} \mathrm{~B}$ nanocrystallites form within the amorphous matrix even in the as-quenched ribbons (figure 1). This is in agreement with the strong chemical short-range order resulting from preferential covalent metal-metalloid bonding, observed in $\mathrm{Fe}-\mathrm{Co}-\mathrm{Si}-\mathrm{B}$ alloys [19]. Upon annealing, the $\mathrm{Co}_{2} \mathrm{~B}$ nanocrystallites may initiate heterogeneous nucleation and perhaps also hinder the occurrence of the glass transition.

The as-quenched and ball-milled samples also differ in their crystallization behaviour. The as-quenched samples exhibit a sharp and intense crystallization peak. In contrast, the enthalpy of the crystallization peak of the powder samples is half the size. This shows that milling caused an extensive crystallization of the as-quenched $\mathrm{Co}_{70.3} \mathrm{Fe}_{4.7} \mathrm{~B}_{25}$ ribbons. The crystallization onset temperatures of the powder and as-quenched samples $T_{x o n s e t}$ are very close, at 735 and $750 \mathrm{~K}$, respectively. The crystallization peak maxima $T_{x}$ are at 781 and $770 \mathrm{~K}$, respectively.

The as-quenched and powder samples after pre-annealing at $893 \mathrm{~K}$ were further examined by high resolution XRD (not shown here). In both cases the XRD patterns are similar (no new and/or vanishing peaks were detected) and the specimens consist of tetragonal $\mathrm{Co}_{2} \mathrm{~B}$ and bcc $\mathrm{Co}_{7} \mathrm{Fe}_{3}$ (SG $\operatorname{Im} \overline{3} m$, PDF no 50-0795) phases.

A small exothermic double peak at $860 \mathrm{~K}$ (marked by the open triangle in the figure inset) is however visible only on the DSC curve of the milled samples. Its existence has been confirmed by repeating the measurement. Based on the XRD experiments we believe its origin is connected to the formation of bcc $\mathrm{Co}_{7} \mathrm{Fe}_{3}$.

\subsection{VSM measurements}

The relatively low value of coercivity $H_{\mathrm{c}}=19 \mathrm{~A} \mathrm{~m}^{-1}$ of as-quenched $\mathrm{Co}_{70.3} \mathrm{Fe}_{4.7} \mathrm{~B}_{25}$ reveals its soft magnetic nature. On the other hand the coercivity of ribbons milled for $2 \mathrm{~h}$ is much higher and shows strong size dependence. As can be seen from table 1, the coercivity of sieved powder fractions increases with decreasing particle size. It is well known that the coercivity of multi-domain particles increases with decreasing size and reaches a maximum when particles reach a critical size and start to behave as a single magnetic domain [20]. The single-domain particle size of $\mathrm{Co}_{1-x} \mathrm{~B}_{x}$ alloys with $0.32 \leqslant x \leqslant 0.4$ is around $d_{\mathrm{s}}=40 \mathrm{~nm}$ (for $\operatorname{Co} d_{\mathrm{s}}=30 \mathrm{~nm}$ ) [21]. The values of saturation magnetization for sieved powder fractions are significantly lower than those of the as-quenched alloy. This is mainly due to the fact that partially crystalline powders exhibit the presence of ferromagnetic $\mathrm{Co}_{2} \mathrm{~B}$, which has a rather low value of saturation magnetization $\left(M_{\mathrm{s}}=73.5 \mathrm{~A} \mathrm{~m}^{2} \mathrm{~kg}^{-1}\right.$ at $4.2 \mathrm{~K}$ [22] $)$ as compared to asquenched $\mathrm{Co}_{70.3} \mathrm{Fe}_{4.7} \mathrm{~B}_{25}$. As indicated by XRD measurements, smaller particles show a higher 


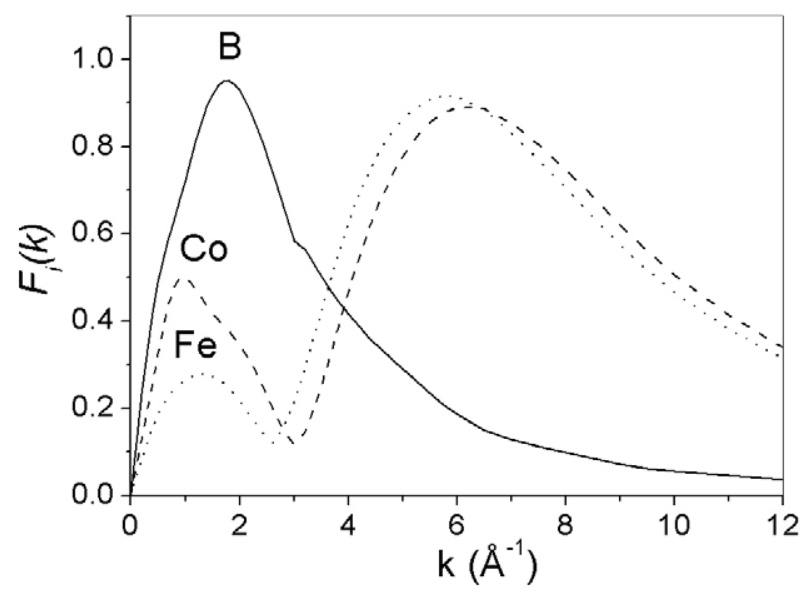

Figure 4. Back-scattering amplitudes (taken from FEFF8.1 code) of atoms located at the following distances from the absorbing atom: Fe and $\mathrm{Co}$ at $2.5 \AA$ and $\mathrm{B}$ at $2 \AA$.

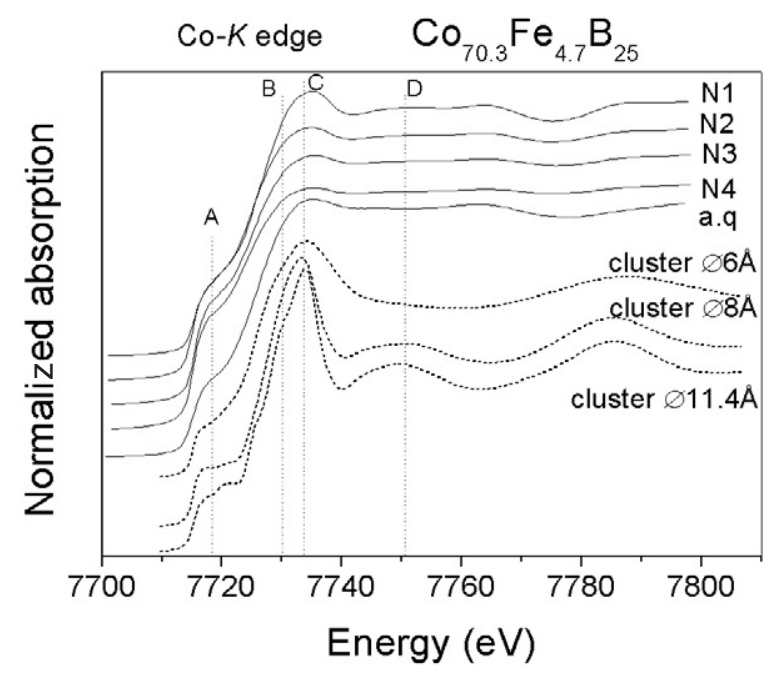

Figure 5. X-ray absorption near $\mathrm{Co} \mathrm{K}$ edge for the $\mathrm{Co}_{70.3} \mathrm{Fe}_{4.7} \mathrm{~B}_{25}$ alloys. Full line, experimental data; dashed lines, theoretical curves. The vertical lines, labelled by letters A, B, C, D, mark distinct features of the XANES structure.

degree of crystallinity and thus higher fraction of $\mathrm{Co}_{2} \mathrm{~B}$. Therefore, slightly higher $M_{\mathrm{S}}$ for smaller powder fractions may seem confusing; however, it reflects the higher weight fraction of Fe-enriched amorphous residual with higher $M_{\mathrm{s}}$, which is embedding $\mathrm{Co}_{2} \mathrm{~B}$ as a result of partial crystallization.

\subsection{XANES}

Figure 4 shows back-scattering amplitudes of B, Co and Fe calculated by the FEFF code. From the figure it follows that for the XANES region (low $k$ range) the back-scattering amplitude of the low-atomic-number B becomes significantly greater than those of the transition metal atoms. Therefore, the XANES is sensitive (more than EXAFS) to the metalloid atom location in the first coordination shell around $\mathrm{Fe}$ and Co. Figures 5 and 6 compare the XANES spectra 


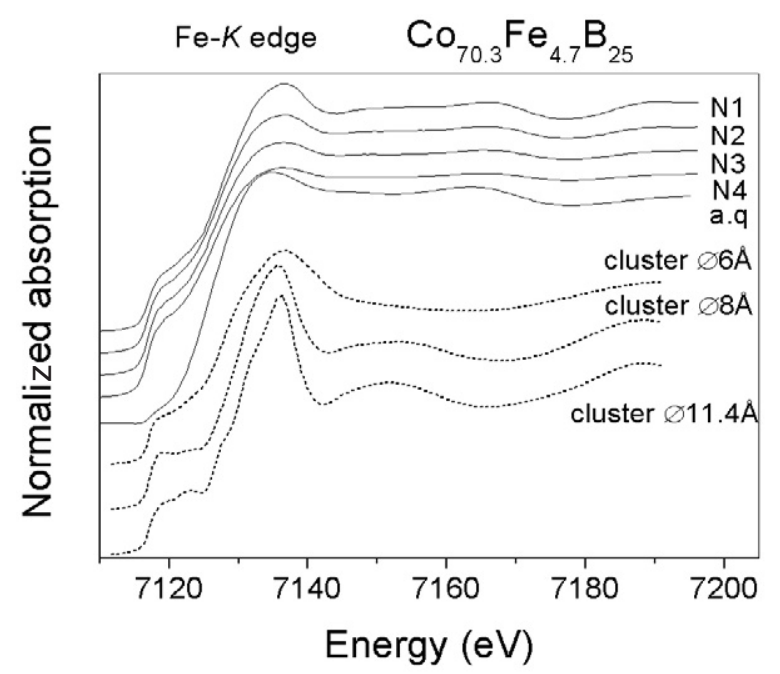

Figure 6. X-ray absorption near $\mathrm{Fe} \mathrm{K}$ edge for the $\mathrm{Co}_{70.3} \mathrm{Fe}_{4.7} \mathrm{~B}_{25}$ alloys, Full line, experimental data; dashed lines, theoretical curves.

measured at the Co and Fe K edges (full curves), respectively. The Fe XANES structures shows the same minima and maxima as those for Co XANES, which means the Co and Fe atoms have very similar local atomic surroundings. The theoretically calculated XANES spectra (dashed curves) can directly be compared to the measured signals and demonstrate how the cluster size influences the corresponding absorption cross-section. Clearly, the XANES features (marked by A, B, C) are dominated by the contribution of the first shell of atoms (cluster diameter $6 \AA$, sketched in figure 2(b)). However, expanding the cluster to the second shell (cluster diameter $8 \AA$ ) is essential in order to reproduce all the features observed in the Co and Fe XANES for the finest (N1, most crystalline) powder sample. On the other hand, adding more shells (cluster diameter $11.4 \AA$ ) to the calculation will result in sharpening of the 'white lines' but does not introduce any other distinct feature.

\subsection{EXAFS}

In order to quantify (not only qualitatively describe) the local atomic environment around $\mathrm{Co}$ and $\mathrm{Fe}$ atoms in as-quenched and milled $\mathrm{Co}_{70.3} \mathrm{Fe}_{4.7} \mathrm{~B}_{25}$, we proceed to analyse the corresponding EXAFS signals. What typically is anticipated in any (unknown structure) EXAFS analysis is a fitting of a reference (known) sample datasets. There are two main reasons for this. (1) Fitting of known structure signal provides information about a possible energy offset (small shift of the measured data on photon energy scale) and instrumental broadening. Especially, the instrumental broadening (which is a function of momentum $k$ always convoluted to the sample signal) could strongly influence the refinement of the coordination number and experimental data must be corrected for it [8]. (2) Further valuable information obtainable from a known structure refinement is an estimate of particular parameter errors. In this manner, the maximal uncertainties of our data were estimated to be $\pm 0.04 \AA$ and \pm 1 atom for interatomic distances and for coordination number, respectively.

The normalized and $k^{2}$ weighted EXAFS data of the as-quenched and powder $\mathrm{Co}_{70.3} \mathrm{Fe}_{4.7} \mathrm{~B}_{25}$ samples measured above the $\mathrm{Co}$ and $\mathrm{Fe} \mathrm{K}$ edges are displayed in figures 7(a) and 8(a), respectively. The EXAFS structure from the as-quenched alloy has a sinusoidal shape 


\section{Co-K edge $\quad \mathrm{Co}_{70.3} \mathrm{Fe}_{4.7} \mathrm{~B}_{25}$}
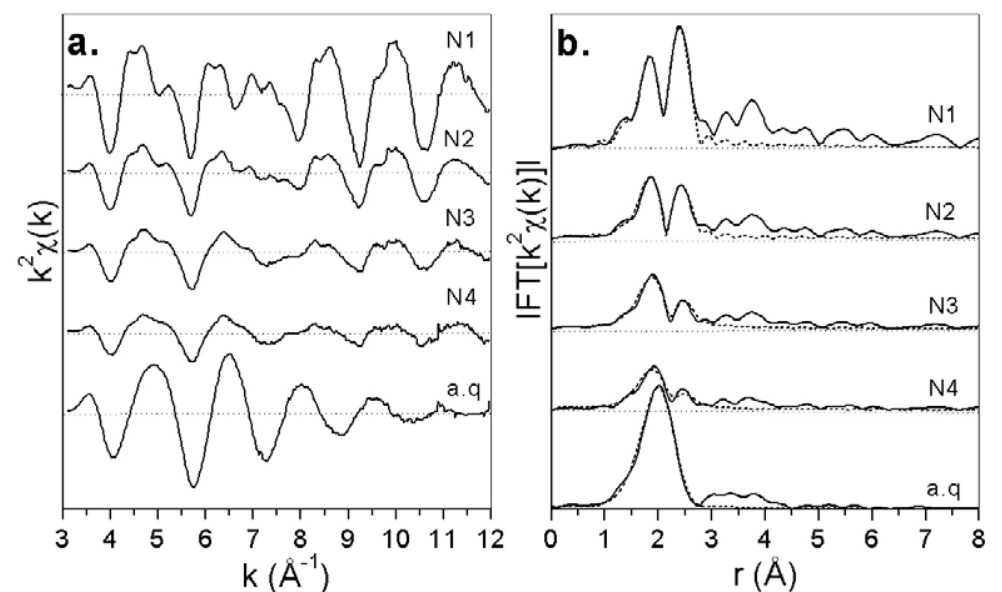

Figure 7. (a) Experimental $k^{2} \chi(k)$ of the $\mathrm{Co}_{70.3} \mathrm{Fe}_{4.7} \mathrm{~B}_{25}$ alloys measured above the Co $\mathrm{K}$ edge; a.q.- - as-quenched (ribbon) sample, sieved powder samples of grain size fractions $<20$ (N1), 2045 (N2), 45-63 (N3) and >63 $\mu \mathrm{m}$ (N4). (b) Corresponding Fourier transforms (full line) shown together with the theoretical model (dashed line) using structural data listed in table 2.

\section{Fe-K edge $\quad \mathrm{Co}_{70.3} \mathrm{Fe}_{4.7} \mathrm{~B}_{25}$}
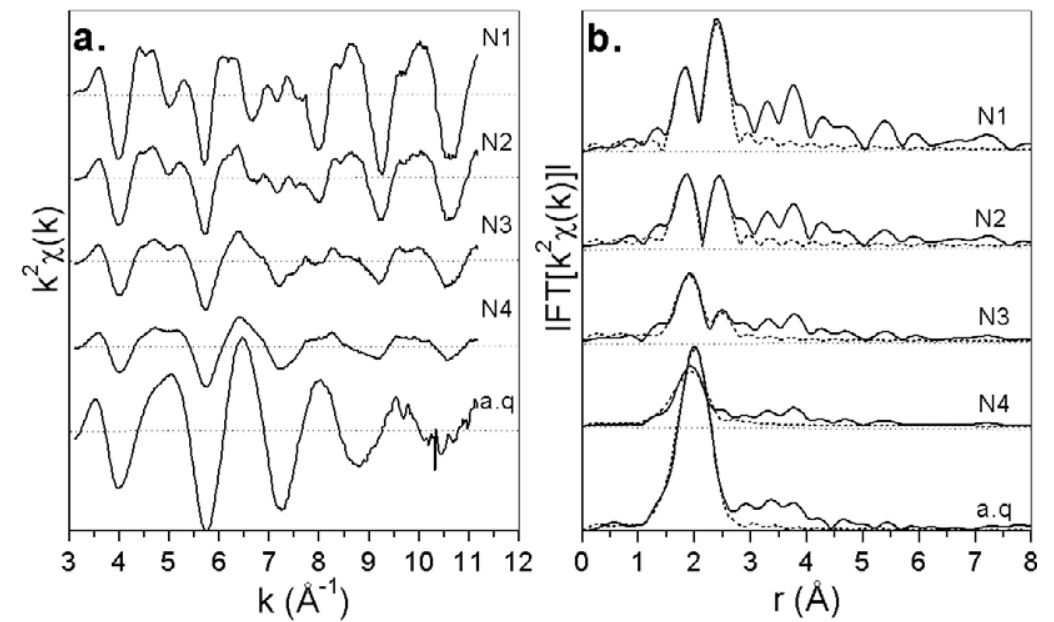

Figure 8. (a) Experimental $k^{2} \chi(k)$ of the $\mathrm{Co}_{70.3} \mathrm{Fe}_{4.7} \mathrm{~B}_{25}$ alloys measured above the $\mathrm{Fe} \mathrm{K}$ edge; a.q.-as-quenched (ribbon) sample, sieved powder samples of grain size fractions $<20$ (N1), 2045 (N2), 45-63 (N3) and >63 $\mu \mathrm{m}$ (N4). (b) Corresponding Fourier transforms (full line) shown together with the theoretical model (dashed line) using structural data listed in table 2.

with an amplitude rapidly decreasing with increasing momentum because of its high structural disorder. Unlike the as-quenched sample, the signals from size fractioned powders show complex beatings differing by large amplitude variations. Detailed inspection of Co and Fe Kedge EXAFS signals taken from the same sample document almost identical oscillations (the same maxima, minima and comparable amplitude), which indicates the local atomic structure around $\mathrm{Co}$ and $\mathrm{Fe}$ atoms is similar. The above statement is fully consistent with our XANES 
observations. Figures 7(b) and 8(b) show corresponding Co and Fe K-edge Fourier transforms (FTs). The plots from the as-quenched sample show in both cases dominant peaks located around $2 \AA$ followed by small wiggles. It was realized that the main peak is a sum of two subshell (B and $\mathrm{Co}$ ) contributions. The FTs from the powder sample, in contrast to the previous one, show two distant maxima. The first one $(\sim 1.9 \AA)$ is a contribution of closest $\mathrm{B}$ atoms while the second $(\sim 2.42 \AA)$ is a sum of two Co coordination shells. The second peak increases substantially with the degree of crystallization. Generally, any EXAFS signal (and its FT, of course) always needs to be interpreted as an interference function, which in an extreme case means that contributions having opposite phases will cancel each other. The intensity of the second peak should be viewed in this light. For instance, in the case of sample N4 the interfering signal from two Co sub-shells (both having a wide spread of inner atoms) gives rise to the small peak only, while the most crystalline sample N1 having localized atoms in the subshells shows stronger interference. The second peak in all cases is visible with a pronounced shoulder on the right side from the peak maximum. Our analysis suggests that it originates from three-path multiple scattering and thus was rejected for the further analysis.

Coming now to the sample structure characterization, one needs to stress here that the EXAFS analysis from ternary alloys often becomes very difficult due to the large number of free parameters (12) involved in the fitting procedure. An effective way to reduce this number-which in consequence will also improve the stability of numerical fitting and reduce undesirable correlations - is the application of additional constraints to the theoretical model. The applied constraints must have both physical and chemical sense, which requires, however, some pre-knowledge about the investigated structure. Some constraints applied in our analysis use previously obtained knowledge mainly from diffraction work, while others reflect some kinds of overall assumption.

- For data from as-quenched sample: only two B and Co shells were assumed, the sum of the atoms in the first coordination shell was constrained to $N_{\mathrm{B}}+N_{\mathrm{Co}}=14$ (the coordination number calculated from XRD); mean square relative displacement and threshold energy shifts were set to be $\sigma_{\mathrm{B}}^{2}=\sigma_{\mathrm{Co}}^{2} ; \Delta E_{0 \mathrm{~B}}=\Delta E_{0 \mathrm{Co}}$.

- For data from powder samples a theoretical model combining three sub-shells, $\mathrm{B}, \mathrm{Co}_{1}$ and $\mathrm{Co}_{2}$, was applied; $N_{\mathrm{B}}+N_{\mathrm{Co} 1}+N_{\mathrm{Co} 2}=15 ; \sigma_{\mathrm{B}}^{2}=\sigma_{\mathrm{Co} 1}^{2}=\sigma_{\mathrm{Co} 2}^{2} ; \Delta E_{0 \mathrm{~B}}=\Delta E_{0 \mathrm{Co} 1}=$ $\Delta E_{0 \mathrm{Co} 2}$.

The reader could raise doubts on the $\sigma_{\mathrm{B}}^{2}=\sigma_{\mathrm{Co}}^{2}$ and $\sigma_{\mathrm{B}}^{2}=\sigma_{\mathrm{Co} 1}^{2}=\sigma_{\mathrm{C} 02}^{2}$ constraints. Presumably, if one expects a strong chemical short-range order between transition metals and boron the $\sigma^{2}$ for TM-B and TM-TM will be different. In order to advocate our constraint it needs to be said that precise quantification of the individual TM-B and TM-TM $\sigma^{2}$ just from the performed experiments is impossible, but these parameters need to be constrained in order to get a unique solution through the fitting. Therefore, the obtained $\sigma^{2}$ parameter from our analysis represents an overall atomic displacement, a term similar to $B_{\text {iso }}$ used for x-ray powder diffraction.

Although the numerical fitting was done on back-transformed $k^{2} \chi(k)$ signals, the results are shown (for clarity reasons on the corresponding FT representations, the dashed lines in figures 7 (b) and $8(\mathrm{~b})$ ). Just visual examination of the plots reveals relatively good model-measurement agreement in as-quenched, N1 and N2 samples, while fitting of the low-crystallinity N3 and N4 samples results in considerable mismatch. The worst fit was achieved in the samples having roughly equal proportions between amorphous and crystalline fractions, which could be explained by a highly asymmetric (non-Gaussian) distribution of back-scattering atoms in these alloys. In fact, EXAFS is very sensitive to the details of the distribution due to the high values of momentum transfer, $Q=2 k$, twice that of a conventional 
diffraction experiment. There are two methods most used for better treatment of disorder effects.

The first one assumes a physically reasonable shape for the non-Gaussian one-dimensional distribution and optimizes its parameters by comparing the experimental EXAFS to the theoretical model. Unfortunately, we do not have the detailed information about the interatomic distribution necessary for this approach in hand. The second method, frequently used by the EXAFS community, is a model-independent approach based on the cumulant expansion of the EXAFS signal. Aside from the fact that this approach will increase the number of free parameters at least by two and despite frequent problems with series convergence, Freund [23] concluded that two additional parameters $\left(C_{3}\right.$ and $\left.C_{4}\right)$ are not statistically justified for the first shell of a selection of metals, semiconductors and halides. For this reason we are satisfied with the simple EXAFS formula, equations (5) and (6), quoted in the theoretical part of this work. The structural parameters refined from our EXAFS signal analysis are listed in table 2, from which we have the following.

- The distance between the Co and B atoms remains (within the instrumental error) constant for all samples investigated; the mean value $\sim 2.15 \AA$ is identical to the one reported for $\mathrm{Co}_{2} \mathrm{~B}$. The separation between the $\mathrm{Fe}$ and $\mathrm{B}$ atoms is, on the other hand, increasing from 2.14 to $2.2 \AA$ depending on sample crystallization. The second coordination shell consisting of Co atoms increases its weighted average separation from the central absorbing $\mathrm{Co}$ and $\mathrm{Fe}$ atoms by 0.11 and $0.08 \AA$, respectively. The value $\sim 2.54 \AA$ is slightly smaller compared to the $2.6 \AA$ reported for crystalline $\mathrm{Co}_{2} \mathrm{~B}$.

- The coordination number obtained from the first shells, reflecting the degree of sample crystallization. For the most crystalline sample, N1, the obtained values are close to the reported $N_{\mathrm{Co}-\mathrm{B}}=4, N_{\mathrm{Co}-\mathrm{Co} 1}=3$ and $N_{\mathrm{Co}-\mathrm{Co} 2}=8$ for $\mathrm{Co}_{2} \mathrm{~B}$.

- The $\sigma^{2}$ values document the progressive decrease of static disorder in the sample containing a larger fraction of crystalline phase; the value from the most crystalline sample $\mathrm{N} 1$ is less than half compared to the as-quenched state. It is also remarkable that the absolute values of $\sigma^{2}$ refined from the Fe EXAFS are smaller in all cases (except the worst fit) compared with the data from the Co K edge; this suggests stronger atomic bonding around $\mathrm{Fe}$ than for $\mathrm{Co}$ atoms. The above observation supports the existence of covalent B atoms bound to central Fe as discussed previously by e.g. Fedez-Gubieda et al for $\mathrm{FeCoSiB}$ [19].

\section{Conclusion}

We have investigated the influence of short-time ball-milling on the atomic structure of amorphous $\mathrm{Co}_{70.3} \mathrm{Fe}_{4.7} \mathrm{~B}_{25}$. For our study two types of samples have been prepared.

(1) Amorphous ribbons, prepared by single-roller melt spinning.

(2) Powder samples, prepared from the ribbons by milling for $2 \mathrm{~h}$ in a planetary ball mill. In order to examine the powder samples in detail, individual size fractions $<20$ (N1), 20-45 (N2), 45-63 (N3) and >63 $\mu \mathrm{m}(\mathrm{N} 4)$ have been isolated from the total volume by sieving.

Structural stability upon annealing was ascertained by DSC measurements while the atomic structure was examined by XRD and Co and Fe K-edge XAFS. The main observations from our study can be summarized in the following items.

(a) The DSC curves from both the ribbons and the powder samples do not show a glass transition. The crystallization enthalpy of the powder sample is less than half compared to 
Table 2. Structural parameters of as-quenched ribbon and powder $\mathrm{Co}_{70.3} \mathrm{Fe}_{4.7} \mathrm{~B}_{25}$ alloy determined from the Co K- and Fe K-edge EXAFS spectra; C and B mean central and back-scattering atom, respectively.

\begin{tabular}{|c|c|c|c|c|c|}
\hline \multirow[b]{2}{*}{ Sample } & \multicolumn{2}{|c|}{ Co K edge } & \multicolumn{2}{|c|}{$\mathrm{Co}_{70.3} \mathrm{Fe}_{4.7} \mathrm{~B}_{25}$} & \multirow[b]{2}{*}{$\Delta E(\mathrm{eV})$} \\
\hline & C-B pair & $R_{\mathrm{C}-\mathrm{B}} \pm 0.04(\AA)$ & $N_{\mathrm{B}} \pm 1$ & $\sigma^{2}\left(\AA^{2}\right) \pm 0.001$ & \\
\hline \multirow[t]{2}{*}{ a.q. } & Co-B & 2.13 & 4.4 & 0.0193 & -15.8 \\
\hline & $\mathrm{Co}-\mathrm{Co}$ & 2.43 & 9.6 & & \\
\hline \multirow[t]{3}{*}{ N1 } & Co-B & 2.16 & 3.7 & 0.0093 & -12.4 \\
\hline & $\mathrm{Co}-\mathrm{Co}_{1}$ & 2.40 & 4.3 & & \\
\hline & $\mathrm{Co}-\mathrm{Co}_{2}$ & 2.63 & 7.0 & & \\
\hline \multirow[t]{3}{*}{$\mathrm{N} 2$} & Co-B & 2.17 & 4.0 & 0.0126 & -13.6 \\
\hline & $\mathrm{Co}-\mathrm{Co}_{1}$ & 2.39 & 5.3 & & \\
\hline & $\mathrm{Co}-\mathrm{Co}_{2}$ & 2.62 & 5.7 & & \\
\hline \multirow[t]{3}{*}{ N3 } & $\mathrm{Co}-\mathrm{B}$ & 2.16 & 4.2 & 0.0163 & -13.3 \\
\hline & $\mathrm{Co}-\mathrm{Co}_{1}$ & 2.40 & 5.7 & & \\
\hline & $\mathrm{Co}-\mathrm{Co}_{2}$ & 2.62 & 5.1 & & \\
\hline \multirow[t]{3}{*}{ N4 } & Co-B & 2.16 & 4.6 & 0.0192 & -14.7 \\
\hline & $\mathrm{Co}-\mathrm{Co}_{1}$ & 2.39 & 5.9 & & \\
\hline & $\mathrm{Co}-\mathrm{Co}_{2}$ & 2.62 & 4.5 & & \\
\hline & \multicolumn{2}{|c|}{ Fe K edge } & \multicolumn{2}{|c|}{$\mathrm{Co}_{70.3} \mathrm{Fe}_{4.7} \mathrm{~B}_{25}$} & \\
\hline Sample & C-B pair & $R_{\mathrm{C}-\mathrm{B}} \pm 0.03(\AA)$ & $N_{\mathrm{B}} \pm 1$ & $\sigma^{2}\left(\AA^{2}\right) \pm 0.001$ & $\Delta E(\mathrm{eV})$ \\
\hline \multirow[t]{2}{*}{ a.q. } & $\mathrm{Fe}-\mathrm{B}$ & 2.14 & 6.2 & 0.0157 & -6.7 \\
\hline & $\mathrm{Fe}-\mathrm{Co}$ & 2.42 & 7.8 & & \\
\hline \multirow[t]{3}{*}{$\mathrm{N} 1$} & $\mathrm{Fe}-\mathrm{B}$ & 2.20 & 3.9 & 0.0078 & -5.1 \\
\hline & $\mathrm{Fe}-\mathrm{Co}_{1}$ & 2.38 & 4.9 & & \\
\hline & $\mathrm{Fe}-\mathrm{Co}_{2}$ & 2.61 & 6.2 & & \\
\hline \multirow[t]{3}{*}{$\mathrm{N} 2$} & $\mathrm{Fe}-\mathrm{B}$ & 2.20 & 4.5 & 0.0103 & -5.1 \\
\hline & $\mathrm{Fe}-\mathrm{Co}_{1}$ & 2.38 & 5.1 & & \\
\hline & $\mathrm{Fe}-\mathrm{Co}_{2}$ & 2.61 & 5.4 & & \\
\hline \multirow[t]{3}{*}{ N3 } & $\mathrm{Fe}-\mathrm{B}$ & 2.18 & 5.3 & 0.0147 & -6.2 \\
\hline & $\mathrm{Fe}-\mathrm{Co}_{1}$ & 2.39 & 5.6 & & \\
\hline & $\mathrm{Fe}-\mathrm{Co}_{2}$ & 2.60 & 4.1 & & \\
\hline \multirow[t]{3}{*}{ N4 } & $\mathrm{Fe}-\mathrm{B}$ & 2.15 & 5.2 & 0.0229 & -6.7 \\
\hline & $\mathrm{Fe}-\mathrm{Co}_{1}$ & 2.41 & 7 & & \\
\hline & $\mathrm{Fe}-\mathrm{Co}_{2}$ & 2.67 & 2.8 & & \\
\hline
\end{tabular}

the equivalent from the as-quenched sample. This indicates crystallization initiated by the milling process.

(b) XRD measurements reveal that the degree of sample crystallinity is inversely proportional to the particle size, which means the finer powder fraction is more crystalline than the coarse one. The crystalline compound present in all samples was identified as a tetragonal phase similar to $\mathrm{Co}_{2} \mathrm{~B}$ (PDF no 25-241; SG $I$ 4 $/ \mathrm{mcm}$ ) having lattice parameters $a=5.06(2)$ and $c=4.19(7) \AA$.

(c) Magnetic properties of different powder fractions were found also be dependent on their particle size.

(d) Both XANES and EXAFS from the same sample reflect very similar local atomic surroundings around the $\mathrm{Co}$ and $\mathrm{Fe}$ atoms. Taking this into account, it is logical to assume that $\mathrm{Fe}$ replaces some $\mathrm{Co}$ atoms in the tetragonal lattice and forms a ternary $(\mathrm{Co}, \mathrm{Fe})_{2} \mathrm{~B}$ 
solid solution which, as has been shown, has lattice parameters slightly different compared to pure $\mathrm{Co}_{2} \mathrm{~B}$.

(e) Calculation of XANES signals proved that clusters of $8 \AA$ diameter containing just two atomic coordination shells are sufficient to reproduce all major features of the experimentally obtained $\mathrm{Co}$ and Fe resonance signals.

(f) The quantitative results from the EXAFS measurements listed in table 2 document the increases of short-range order with decreasing powder particle size. The existence of covalent bonding between $\mathrm{Fe}$ and $\mathrm{B}$ in our $\mathrm{Co}_{70.3} \mathrm{Fe}_{4.7} \mathrm{~B}_{25}$ alloy is very likely.

Our results show that fine powder fractions are the ones which are the most affected by ballmilling. The degree of crystallinity and magnetic properties of different powder fractions were found to depend on their particle size. Recently, we have shown that compaction of $\mathrm{Co}_{70.3} \mathrm{Fe}_{4.7} \mathrm{~B}_{25}$ powder using uniaxial pressure of $900 \mathrm{MPa}$ for $2 \mathrm{~min}$ at a temperature of $460^{\circ} \mathrm{C}$ leads to a bulk material with DC coercivity of $2400 \mathrm{~A} \mathrm{~m}^{-1}$, which can be further lowered to $400 \mathrm{~A} \mathrm{~m}^{-1}$ by compaction of cryomilled ribbons [15]. On the other hand, it was reported in [24] that the presence of a small fraction of crystalline phase (1-5\%) improves the AC magnetic properties at higher temperatures. We conclude that ball-milling of amorphous precursor represents a practical way to prepare amorphous/nanocrystalline composite materials, which can be after proper selection of powder fractions consolidated into bulk material having the desired AC magnetic properties, which will be a subject of the next studies.

\section{References}

[1] McHenry M E, Willard M A and Laughlin D E 1999 Prog. Mater. Sci. 44291

[2] Zarzycki J 1991 in Glasses and Amorphous Materials (Mater. Sci. Technol. vol 9) ed J Zarzycki (Weinheim: VCH-Verlag) p 91

[3] Bednarcik J, Kollar P, Roth S and Eckert J 2003 Phys. Status Solidi a 199299

[4] Roth S, Ferchmin A R and Kobe S 1994 Numerical Data and Functional Relationships in Science and Technology (Landolt-Bornstein vol III/19) (Berlin: Springer) p 144

[5] Makino A, Hatanai T, Naitoh Y, Bitoh T, Inoue A and Masumoto T 1997 IEEE Trans. Magn. 333793

[6] Hammersley A P, Svensson S O, Hanfland M, Fitch A N and Häusermann D 1996 High Pressure Res. 14235

[7] Faber T E and Ziman J M 1965 Phil. Mag. 11153

[8] Ankudinov A L, Ravel B, Rehr J J and Conradson S D 1998 Phys. Rev. B 587565

[9] Klementiev K V VIPER for Windows, freeware www.desy.de/ klmn/viper.html

Klementev K V 2001 J. Phys. D: Appl. Phys. 34209

[10] Benfatto M, Natoli C R, Bianconi A, Garcia J, Marcelli A, Fanfoni M and Davoli I 1986 Phys. Rev. B 345774

[11] Mueller J E, Jepsen O, Andersen O K and Wilkins J W 1975 Phys. Rev. Lett. 40720

[12] Durham P J 1988 X-Ray Absorption (New York: Wiley) p 53

[13] Zhang X G and Gonis A 1989 Phys. Rev. Lett. 621161

[14] Teo B K 1986 EXAFS: Basic Principles and Data Analysis (Berlin: Springer) p 64

[15] Füzer J, Bednarcik J, Kollar P and Roth S 2006 J. Magn. Magn. Mater. doi:10.1016/j.jmmm.2006.11.014

[16] Havinga E E, Damsma H and Hokkeling P 1972 J. Less-Common Met. 27169

[17] Saksl K, Jóvári P, Franz H and Jiang J Z 2005 J. Appl. Phys. 97113507

[18] Saksl K, Jóvári P, Franz H, Zeng Q S, Liu J F and Jiang J Z 2006 J. Phys.: Condens. Matter 187579

[19] Fdez-Gubieda M L, Orúe I, Plazaola F and Barandiarán J M 1996 Phys. Rev. B 53620

[20] Cullity B D 1972 Introduction to Magnetic Materials (Reading, MA: Addison-Wesley)

[21] Yiping L, Hadjipanayis G C and Sorensen C M 1989 J. Magn. Magn. Mater. 79321

[22] Kanomata T et al 1997 J. Alloys Compounds 259 L1

[23] Freund J 1991 Phys. Lett. A 157256

[24] Shaikh A K, Wexler D and Delamore G W 1996 J. Magn. Magn. Mater. 152345 